\title{
Selected recent HERMES results on parton distribution and fragmentation functions
}

\author{
Klaus RITH $^{* \dagger}$ (on behalf of the HERMES collaboration) \\ University of Erlangen-Nürnberg and DESY \\ E-mail: klaus.rithedesy.de
}

The HERMES experiment has studied for more than a decade deep-inelastic scattering of electrons/positrons from longitudinally and transversely polarised and also unpolarised atomic gas targets internal to the electron storage ring of HERA. The collected semi-inclusive data allowed to extract informations about various quark distribution and fragmentation functions. Examples presented in this contribution are: a novel determination of the unpolarised strange-quark (plus anti-quark) distribution; the measurement of single-spin azimuthal asymmetries for pions and kaons from a transversely polarised hydrogen target that are related to the quark transversity distribution in conjunction with the spin-dependent Collins fragmentation function and also to the Sivers distribution function; and the measurement of azimuthal hadron asymmetries in unpolarised deep-inelastic scattering related to the so-called Cahn effect and the Boer-Mulders distribution function.

European Physical Society Europhysics Conference on High Energy Physics

July 16-22, 2009

Krakow, Poland

\footnotetext{
${ }^{*}$ Speaker.

${ }^{\dagger}$ Supported by the German BMBF - contract nr. 06 ER 143
} 


\section{Introduction}

A complete description of the partonic structure of the nucleon in leading twist requires three quark distribution functions (DFs) that survive integration over intrinsic transverse momenta. These are the unpolarised quark DF $q\left(x, Q^{2}\right)$, the quark helicity DF $\Delta q\left(x, Q^{2}\right)$, and the chiral-odd transversity DF $\delta q\left(x, Q^{2}\right)$ [1]. In addition there are five other transverse-momentum dependent DFs that do not survive the integration [2]. Experimentally these are essentially unexplored. Examples are the time-reversal odd Sivers DF [3], $f_{1 T}^{\perp}\left(x, Q^{2}\right)$, that describes the distribution of unpolarised quarks in a transversely polarised nucleon and can be related to orbital angular momenta of quarks [4], and the Boer-Mulders DF [5], $h_{1}^{\perp}\left(x, Q^{2}\right)$, for transversely polarised quarks in an unpolarised nucleon. Information about these DFs can be obtained from semi-inclusive deep-inelastic scattering (SIDIS) of electrons or positrons from nucleons. In Leading Order (LO) the SIDIS cross section for production of a hadron of type $h$ takes the factorized form

$$
\sigma^{e N \rightarrow e h X}=\sum_{q} D F^{N \rightarrow q}\left(x, Q^{2}\right) \otimes \sigma^{e q \rightarrow e q}\left(x, Q^{2}\right) \otimes F F^{q \rightarrow h}\left(z, Q^{2}\right) .
$$

The sum is over quark and antiquark flavors $q=(u, \bar{u}, d, \bar{d}, s, \bar{s}, \ldots)$, the distribution function $D F^{N \rightarrow q}$ is the quark number density in the nucleon, $\sigma^{e q \rightarrow e q}$ is the hard electron-quark scattering cross section, and the fragmentation function $F F^{q \rightarrow h}$ is a measure of the probability that a quark of flavor $q$ will fragment into a hadron of type $h$. Furthermore $x$ is the Bjorken scaling variable which can be interpreted as the fraction of nucleon momentum carried by the struck quark in the infinitemomentum frame, $z$ is the fractional energy of the virtual photon carried by the produced hadron in the laboratory system and $-Q^{2}$ is the squared four-momentum transfer. Examples for fragmentation functions are the unpolarised FF $D_{1, q}$ and the chiral-odd polarised Collins FF $H_{1, q}^{\perp}$ [6].

\section{The HERMES experiment}

HERMES is one of the three experiments at the HERA electron-proton collider that took data until mid 2007, when the accelerator complex was shut down. It used the high-current longitudinally polarised electron/positron beam of HERA with an energy of $E=27.6 \mathrm{GeV}$ together with polarised and unpolarised gas targets internal to the storage ring. Scattered electrons and particles produced in the deep-inelastic lepton-nucleon interactions were detected and identified by an opengeometry forward spectrometer [7] with large momentum and solid-angle acceptance. The primary scientific goal of HERMES was the detailed investigation of the spin-structure of the nucleon. From the precise measurement of the polarised deuteron structure function $g_{1}^{d}$ the contribution of quark spins, $\Delta \Sigma$, to the spin of the nucleon was determined in NLO-QCD and in the MS-bar scheme to be [8]: $\Delta \Sigma=0.330 \pm 0.025$ (stat) \pm 0.030 (sys). Precise informations about the flavor-separated quark (anti-quark) helicity distribution functions $\Delta q\left(x, Q^{2}\right)$ have been obtained from double-spin asymmetries for various identified hadrons in semi-inclusive polarised deep-inelastic scattering [9]. But the physics reach of this experiment is well beyond this specific aspect of hadron physics and the experiment can be considered as a facility to explore many details of hadron structure, hadron production and hadronic interactions with electromagnetic probes at centre-of-mass energies of around $7 \mathrm{GeV}$. In this contribution HERMES measurements are presented that provide novel information on various quark distribution and fragmentation functions. 

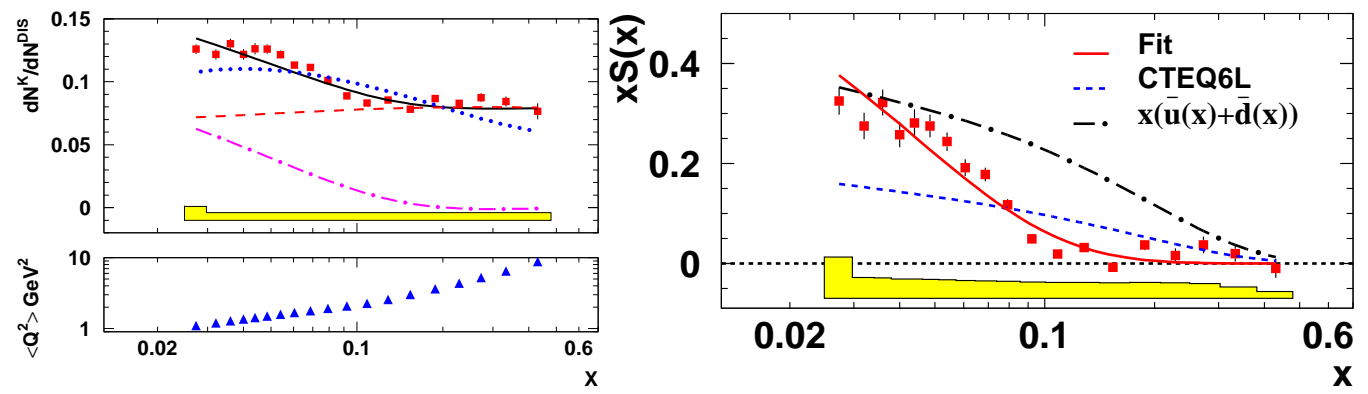

Figure 1: HERMES results for the multiplicity of charged kaons in semi-inclusive DIS from a deuterium target (left panel) and of the derived strange parton distribution $x S(x)$ at $Q_{0}^{2}=2.5 \mathrm{GeV}^{2}$ (right panel), as a function of Bjorken $x$.

\section{The strange quark distribution function}

The experimental information about the distribution function $s(x)(\bar{s}(x))$ of strange quarks (antiquarks) as a function of $x$ is surprisingly scarce. Most of the experimental constraints are based on measurements of oppositely charged muon pairs in deep-inelastic neutrino and antineutrino scattering. In absence of significant experimental constraints, most of the current global QCD fits of parton distribution functions (PDFs) assume $s(x)(\bar{s}(x))$ to be related to the DFs of light antiquarks by $s(x)=\bar{s}(x)=r[\bar{u}(x)+\bar{d}(x)] / 2$ with $r \approx 0.3-0.5$ at some low factorisation scale. HERMES has recently performed the first extraction of $S(x)=s(x)+\bar{s}(x)$ from the multiplicity of charged kaons in semi-inclusive deep-inelastic scattering from a deuteron target [10]. Because strange quarks carry no isospin, the strange seas in the proton and the deuteron can be assumed to be identical. In the deuteron, an isoscalar target, the fragmentation process in deep-inelastic scattering can be described by fragmentation functions that have no isospin dependence. Aside from isospin symmetry between proton and neutron, the only symmetry assumed is charge-conjugation invariance in fragmentation. In LO the charged kaon multiplicities are then given by

$$
\frac{d N^{K}(x)}{d N^{D I S}(x)}=\frac{Q(x) \int D_{1, Q}^{K}(z) d z+S(x) \int D_{1, S}^{K}(z) d z}{5 Q(x)+2 S(x)}
$$

Here $Q(x) \equiv u(x)+\bar{u}(x)+d(x)+\bar{d}(x), D_{1, Q}^{K}(z) \equiv 4 D_{1, u}^{K}(z)+D_{1, d}^{K}(z)$ and $D_{1, S}^{K}(z) \equiv 2 D_{1, s}^{K}(z)$, and $z \equiv E_{K} / v$ with $v$ and $E_{K}$ the energies of the virtual photon and the detected kaon in the target rest frame. The measured kaon multiplicity corrected to $4 \pi$ is shown in the left panel of Fig. 1 as a function of $x$. The data are not reproduced (see dotted curve) by fitting the points using the CTEQ6L [11] strange quark DFs and with $\int D_{1, Q}^{K}(z) d z$ and $\int D_{1, S}^{K}(z) d z$ as free parameters. Instead $\int_{0.2}^{0.8} D_{1, Q}^{K}(z) d z=0.398 \pm 0.010$ was determined from the data at $x>0.15$, where $S(x)$ is compatible with zero. This value was then used together with values of $Q(x)$ from CTEQ6L and the value $\int D_{1, S}^{K}(z) d z=1.27 \pm 0.13$ from de Florian et al. [12] to obtain in an iterative procedure the distribution $x S(x)$ presented in the right panel of Fig. 1. Hereby the multiplicities were evolved to a common $Q_{0}^{2}=2.5 \mathrm{GeV}^{2}$. The solid curve is a fit to the data. The shape is incompatible with $x S(x)$ from CTEQ6L as well as the assumption of an average of an isoscalar nonstrange sea. 


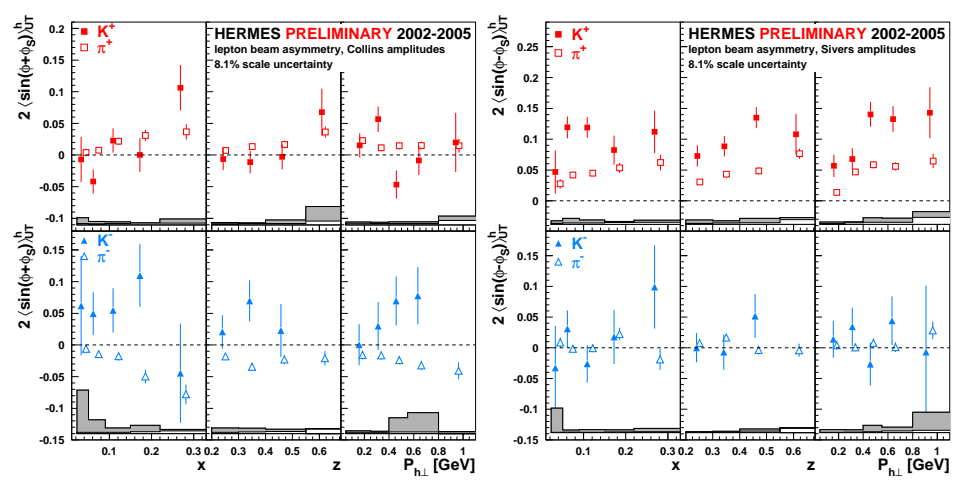

Figure 2: HERMES results for the Collins moments (left panel) and the Sivers moments (right panel) for charged pions and kaons obtained with a transversely polarised hydrogen target.

\section{Transversity, Collins and Sivers Effect}

So far very little experimental information exists about the transversity $\mathrm{DF} \delta q\left(x, Q^{2}\right)$. It is a chiral-odd quantity and cannot be probed in inclusive DIS since hard interactions conserve chirality. Its measurement requires processes involving some additional chiral-odd structure like, e.g., another transversity distribution in transversely polarised Drell-Yan production of lepton pairs, or a chiral-odd fragmentation function in SIDIS. One of the possibilities to study transversity is via the azimuthal angular asymmetry in the distribution of hadrons produced in SIDIS from a transversely polarised target. Such an asymmetry can arise if the transverse polarization of the struck quark influences the transverse momentum of the produced hadron and thereby its distribution in the azimuthal angle $\phi$ about the virtual photon direction relative to the lepton scattering plane. The corresponding chiral-odd fragmentation function $H_{1, q}^{\perp}$ that is known as the Collins FF is also odd under naive time reversal.

Such an angular asymmetry could, however, also be produced by a different mechanism involving correlations between the transverse polarization of the target nucleon and the transverse momentum of quarks. The transverse quark momentum can survive both the photo-absorption and the fragmentation and can reappear in the transverse momentum of the produced hadron and thereby influence its azimuthal angular distribution. The corresponding Sivers DF $f_{1 T}^{\perp}$ is naivetime-reversal odd. Its measurement therefore requires initial or final state interactions. One especially interesting aspect of this distribution function is the possible relation to orbital angular momenta of quarks [4].

Measurements with a transversely polarised target allow to distinguish between the Collins mechanism and the Sivers mechanism. The Collins (Sivers) mechanism will cause a $\sin \left(\phi+\phi_{S}\right)$ $\left(\sin \left(\phi-\phi_{S}\right)\right)$ moment proportional to a convolution of $\delta q(x)$ and $H_{1, q}^{\perp}(z)\left(f_{1 T, q}^{\perp}(x)\right.$ and $\left.D_{1, q}(z)\right)$. Here $\phi_{S}$ is the azymuthal angle between the electron scattering plane and the target spin axis.

Preliminary HERMES results [13] for the Collins and Sivers moments for charged pions and kaons, obtained from data taken in the years 2002-2005 with a transversely polarised hydrogen target, are shown in Fig. 2. The measured Collins asymmetries (left panel) are small but different from zero providing evidence for the existence of both $\delta q(x)$ and $H_{1, q}^{\perp}(z)$. The large $\pi^{-}$moment indicates that the unfavored Collins FF has similar magnitude as the favored one, but opposite sign. 

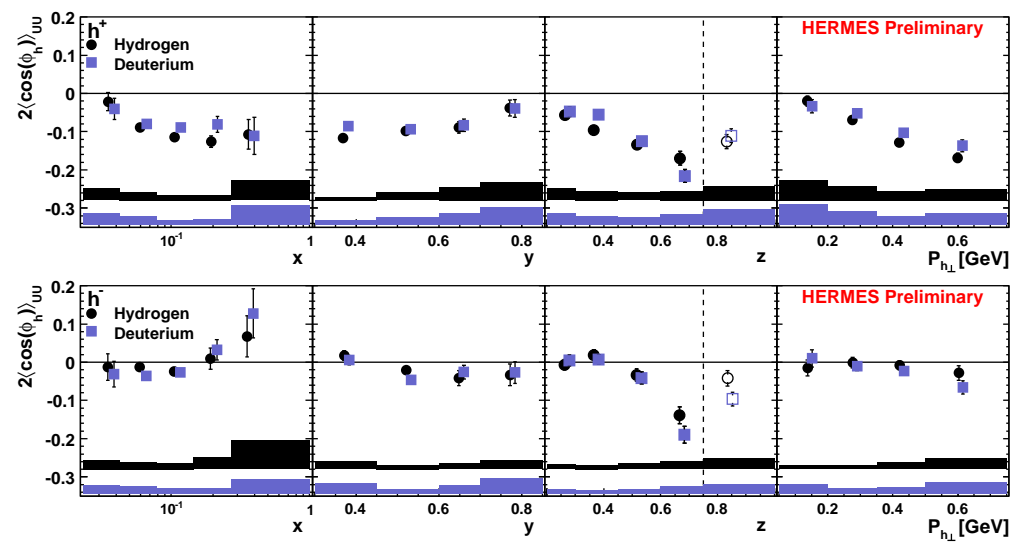

Figure 3: $\cos \phi$ moments for positive (upper panel) and negative (lower panel) hadrons, extracted from hydrogen (circles) and deuterium (squares) data, shown as projections versus the kinematic variables $x, y, z$ and $P_{h \perp}$.

The preliminary results for the $\pi^{+}$and $K^{+}$Sivers asymmetries (upper row of the right panel) are significantly positive, providing the first evidence for a T-odd PDF appearing in leptoproduction. The final results for the Sivers asymmetries [14] are very similar. Consequently one has to conclude from this result that orbital angular momenta of quarks inside the nucleon are non-zero. At present it is, however, not jet possible to quantitatively relate the magnitude of this asymmetry to the fraction of nucleon spin which can be attributed to orbital angular momenta of quarks. The positive kaon amplitudes appear to be larger than the pion amplitudes, which might point to a large Sivers function for sea-quarks. These measurements are an important input for an extraction of the transversity DF, the Collins FF, and the Sivers DF from world data [15].

\section{Cahn and Boer-Mulders effect}

If the semi-inclusive unpolarised DIS cross section is unintegrated over the hadron momentum component transverse to the virtual photon direction, $P_{h \perp}$, an azimuthal dependence around the virtual photon direction exists, which has a $\cos \phi$ and a $\cos 2 \phi$ component. Two mechanisms are expected to give important contributions to this azimuthal dependence: the Cahn effect, a pure kinematic effect, generated by the non-zero intrinsic transverse motion of quarks [16] and the BoerMulders effect, which originates from a coupling between quark transverse momentum and quark transverse spin. To extract the $\cos \phi$ and $\cos 2 \phi$ modulations from the unpolarised HERMES hydrogen $(\mathrm{H})$ and deuterium (D) data a multi-dimensional unfolding procedure was used which takes into account radiative and detector smearing, and in which the event sample is binned simultaneously in the relevant kinematic variables $x, z, P_{h \perp}$ and $y=v / E$. The preliminary $\cos \phi$ moments from the $\mathrm{H}$ and $\mathrm{D}$ data [17] are shown in Fig. 3 as projections versus the four variables. Corresponding data exist for the $\cos 2 \phi$ moments. Both $\mathrm{H}$ and $\mathrm{D}$ data show similar behaviour. $\cos \phi$ moments receive contributions from both the product of the Boer-Mulders DF and the Collins FF, $h_{1, q}^{\perp}(x) \cdot H_{1, q}^{\perp}(z)$, and the product of the unpolarised DF and the normal unpolarised FF, $q(x) \cdot D_{1, q}(z)$. They are found to be sizable and negative for positive hadrons, the signal for negative hadrons is significantly lower. The $\cos 2 \phi$ moments are proportional to $h_{1, q}^{\perp}(x) \cdot H_{1, q}^{\perp}(z)$. They are found to be slightly negative for 
positive hadrons and slightly positive for negative hadrons in agreement with models which predict opposite Boer-Mulders contributions for differently charged hadrons.

\section{References}

[1] V. Barone and P.G. Ratcliffe, Transverse Spin Physics, World Scientific, Singapore 2003.

[2] P. J. Mulders and R. D. Tangerman, The Complete tree level result up to order $1 / Q$ for polarized deep inelastic leptoproduction, Nucl. Phys. B461 (1996) 197; Erratum Nucl. Phys. B484 (1997) 538.

[3] D. W. Sivers, Single Spin Production Asymmetries from the Hard Scattering of Point-Like Constituents, Phys. Rev. D 41(1990) 83.

[4] M. Burkardt, Impact parameter dependent parton distributions and transverse single spin asymmetries, Phys. Rev. D 66 (2002) 114005, [hep-ph/020 9179].

[5] D. Boer and P.J. Mulders, Time-reversal odd distribution functions in leptoproduction, Phys. Rev. D 57 (1998) 5780, [hep-ph / 9711485$].$

[6] J. Collins, Fragmentation of transversely polarized quarks probed in transverse momentum distributions, Nucl. Phys. B 396 (1993) 161, [hep-ph/9208213].

[7] K. Ackerstaff et al.(HERMES), The HERMES spectrometer Nucl. Instrum. Methods A 417 (1998) 230.

[8] A. Airapetian et al.(HERMES), Precise determination of the spin structure function g1 of the proton, deuteron and neutron Phys. Rev. D 75 (2007) 012007 [hep-ex/ 0609039 ].

[9] A. Airapetian et al.(HERMES), Quark helicity distributions in the nucleon for up, down, and strange quarks from semi-inclusive deep-inelastic scattering Phys. Rev. D 71 (2005) 012003 [hep-ex/0407032].

[10] A. Airapetian et al.(HERMES), Measurement of Parton Distributions of Strange Quarks in the Nucleon from Charged-Kaon Production in Deep-Inelastic Scattering on the Deuteron, Phys. Lett. B 666 (2008) 466 [arXiv: 0803.2993$].$

[11] J. Pumplin et al., New generation of parton distributions with uncertainties from global QCD analysis, JHEP02077 (2002) 012 [hep-ph / 0201195$].$

[12] D. de Florian et al., Global analysis of fragmentation functions for pions and kaons and their uncertainties Phys. Rev. D 75(2007) 114010 [hep-ph/ 0703242].

[13] M. Diefenthaler (for the HERMES collaboration), HERMES measurements of Collins and Sivers asymmetries from a transversely polarised hydrogen target, in proceedings of 15 th International Workshop on Deep-Inelastic Scattering (DIS2007) 579, [arXiv : 0706.2242$].$

[14] A. Airapetian et al.(HERMES), Observation of the Nä̈ve-T-odd Sivers Effect in Deep-Inelastic Scattering, [arXiv:0906.3918].

[15] M. Anselmino et al., Sivers and Collins Effects: from SIDIS to Proton-Proton Inclusive Pion Production, [arXiv:0907.3999.

[16] R.N. Cahn, Azimuthal Dependence In Leptoproduction: A Simple Parton Model Calculation, Phys. Lett. B 78 (1978) 269.

[17] F. Giordano and R. Lamb, Measurement of Azimuthal Asymmetries of the Unpolarized Cross-Section at HERMES, in proceedings of 18th International Spin Physics Symposium (Spin2008) AIP Conference Proceedings1149 (2009) 423. 